University of New Mexico

UNM Digital Repository

Mathematics and Statistics Faculty and Staff

Publications

Academic Department Resources

$12-2020$

Structure, NeutroStructure, and AntiStructure in Science

Florentin Smarandache

Follow this and additional works at: https://digitalrepository.unm.edu/math_fsp

Part of the Epistemology Commons, Logic and Foundations Commons, Other Applied Mathematics Commons, Other Mathematics Commons, Other Philosophy Commons, and the Set Theory Commons 


\title{
Structure, NeutroStructure, and AntiStructure in Science
}

\author{
Florentin Smarandache \\ University of New Mexico \\ Mathematics, Physical and Natural Science Division \\ 705 Gurley Ave., Gallup, NM 87301, USA \\ E-mail: smarand@unm.edu
}

\begin{abstract}
In any science, a classical Theorem, defined on a given space, is a statement that is $100 \%$ true (i.e. true for all elements of the space). To prove that a classical theorem is false, it is sufficient to get a single counter-example where the statement is false.

Therefore, the classical sciences do not leave room for partial truth of a theorem (or a statement). But, in our world and in our everyday life, we have many more examples of statements that are only partially true, than statements that are totally true.

The NeutroTheorem and AntiTheorem are generalizations and alternatives of the classical Theorem in any science.

More general, by the process of NeutroSophication, we have extended any classical Structure, in no matter what field of knowledge, to some NeutroStructure, and by the process of AntiSophication to some AntiStructure.
\end{abstract}

Keywords : Structure, NeutroStructure, and AntiStructure

1. The Neutrosophic Triplet $(<A>,<$ neut $A>$, $<$ antiA $>)$

Let $S$ be a given non-empty space (or set) from a universe of discourse $U$.

In neutrosophy, the general neutrosophic triplet $(\langle A\rangle,<$ neut $A\rangle,<$ anti $A\rangle)$, sometimes using the notation $<$ neutro $A>$ for the middle term, can be written as:

$(\langle A(1,0,0)\rangle,\langle A(T, I, F)\rangle,\langle A(0,0,1)\rangle)$, where $(T, I, F) \notin\{(1,0,0),(0,0,1)\} ;$

i.e. $A(1,0,0)$ means that $<\mathrm{A}>$ is $100 \%$ true $(\mathrm{T}=1), 0 \%$ indeterminate $(\mathrm{I}=0)$, and $0 \%$ false $(\mathrm{F}=0)$;

$A(T, I, F)$ means that $<\mathrm{A}>$ is $\mathrm{T} \%$ true, $\mathrm{I} \%$ indeterminate, and $\mathrm{F} \%$ false, where $(\mathrm{T}, \mathrm{I}, \mathrm{F}) \notin\{(1,0,0),(0,0,1)\}$; respectively.

and $A(0,0,1)$ means that $<\mathrm{A}>$ is $0 \%$ true $(\mathrm{T}=0), 0 \%$ indeterminate $(\mathrm{I}=0)$, and $100 \%$ false $(\mathrm{F}=1)$,

\section{Example of Neutrosophic Triplet when $<A>=$ Operation} means:

In the case when $<\mathrm{A}>$ is an Operation (or Operator, Function, Law), on the given space $S$, then $<\mathrm{A}(\mathrm{T}, \mathrm{I}, \mathrm{F})>$

Operation $<\mathrm{A}>$ is $\mathrm{T} \%$ well-defined (or inner-defined, inside of $\mathrm{S}$ ),

$\mathrm{I} \%$ indeterminate-defined (undefined, unknown), and $\mathrm{F} \%$ outer-defined (outside of S).

\section{Neutrosophic Triplet Concepts}

We have the following particular neutrosophic triplets of notions defined on $S$ : 


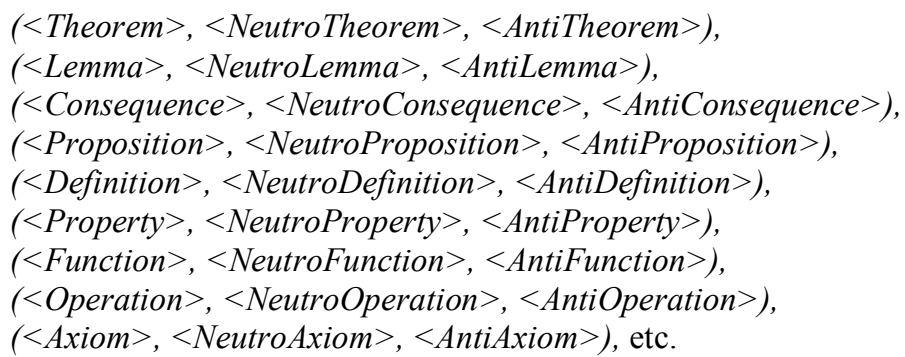

These neutrosophic triplets are referred to any field of knowledge, not only to mathematics.

\section{Theorem, NeutroTheorem, AntiTheorem}

Let's take the first neutrosophic triplet:

$(<$ Theorem $>$, $<$ NeutroTheorem $>$, $<$ AntiTheorem $>$ ).

For the other neutrosophic triplets, it will be similar.

Let $T, I, F \in[0,1]$ be single-valued numbers representing respectively the degree of truth $(\mathrm{T})$, degree of indeterminacy (I), and degree of falsehood (F).

(i) A classical Theorem is a statement that is true (T) for all elements of the space $\mathrm{S}$. Therefore, (T, I, $\mathrm{F})=(1,0,0)$

(ii) A NeutroTheorem is a statement that is partially (T), partially indeterminate (I), and partially false (F), where $(T, I, F) \notin\{(1,0,0),(0,0,1)\}$;

(iii) An AntiTheorem is a statement that is false (F) for all elements of the space $S$. Therefore, (T, I, F) = $(0,0,1)$.

We can rewrite this neutrosophic triplet as:

$(\langle$ Theorem $(1,0,0)\rangle,\langle$ Theorem $(T, I, F)\rangle,\langle$ Theorem $(0,0,1)\rangle)$,

where $(T, I, F) \notin\{(1,0,0),(0,0,1)\}$.

Let T, I, F be intervals (and in general any subsets) from $[0,1]$.

If a Theorem is, let's say, between $90 \%-100 \%$ true, i.e. Theorem( $[0.9,1], 0,0)$, it does not satisfy the classical Theorem $(1,0,0)$, since there is some uncertainty (unclearness) with respect to its degree of truth $[0.9,1] \neq 1$. So, this case goes under NeutroTheorem.

If the Theorem is, let's say again, between $99 \%-100 \%$ false, i.e. Theorem $(0,0,[0.99,1])$, it does not satisfy the AntiTheorem, since similarly there is some uncertainty (unclearness) with respect to its degree of falsehood [0.99, $1] \neq 1$. This case goes also under NeutroTheorem.

In conclusion, no matter if T, I, F are single-valued numbers, intervals, and in general any subsets of $[0,1]$, the neutrosophic triplet of each concept is the same.

Classical Theorem $=<$ Theorem $(1,0,0)>$

NeutroTheorem $=<$ Theorem $(T, I, F)>$, with $(T, I, F) \notin\{(1,0,0),(0,0,1)\}$;

AntiTheorem $=<$ Theorem $(0,0,1)>$.

\section{Remark}

Let $T, I, F \in[0,1]$ and an axiom $\langle A(T, I, F)\rangle$, which means that the axiom has the neutrosophic degree of truth $T$, the neutrosophic degree of indeterminacy $I$, and the neutrosophic degree of falsehood $F$.

If $I>0$ or $0<F<1$, the $\langle A\rangle$ is a NeutroAxiom.

Proof:

If $I>0$, then $(T, I, F) \notin\{(1,0,0),(0,0,1)\}$, because the last two neutrosophic triplets have both $I=0$. 
If $0<F<1$, then again $(T, I, F) \notin\{(1,0,0),(0,0,1)\}$ because the last two neutrosophic triplets have $F=$ 0 and respectively $F=1$.

\section{Elementary Examples of NeutroConcepts and AntiConcepts}

\subsection{NeutroOperation}

Let $Z$ be the set of integers, and $Q$ the set of rational numbers that is considered the universal set, with $Z \subset Q$. Let's define the operation of division:

This is a NeutroDivision because:

$$
\div: Z \times Z \rightarrow Z
$$

(i) There exist the integers $15,5 \in \mathbb{Z}$ such that $15 \div 5=3 \in Z$; this is degree of well-defined (inner-defined);

(ii) There exist the integers $7,0 \in \mathbb{Z}$ such that $7 \div 0=$ undefined; this is degree of indeterminacy; defined.

(iii) There exist the integers $11,2 \in \mathbb{Z}$ such that $11 \div 2=5.5 \notin Z$ or $5.5 \in Q \backslash \mathbb{Z}$; this is degree of outer-

\subsection{AntiOperation}

Let $Z^{-}=\{-1,-2,-3, \ldots,-\infty\}$ the set of negative integers,

$\mathbb{C}=\{a+b i ; a, b \in \mathbb{R}, i=\sqrt{-1}\}$ the set of complex numbers that acts as the universal set of $Z^{-}$.

Let's define the operation of square $\operatorname{root}(\sqrt{ })$ :

$\sqrt{ }: Z^{-} \rightarrow Z^{-}$.

For any negative integer $-a$, where $a>0$ is a positive integer,

$\sqrt{-a}=i \sqrt{a} \notin \mathbb{Z}^{-}$, or $\sqrt{-a} \in C-\mathrm{Z}$.

Therefore the operation $\sqrt{ }$ is totally outer-defined.

\subsection{NeutroFunction} function:

On the set of integers $\mathbb{Z}$ and its universe of discourse $Q$, which is the set of rational numbers, we define the

$$
f: \mathbb{Z} \rightarrow \mathbb{Z}, f(x)=\frac{12}{x} .
$$

(i) There exists the integer, for example $x=6$, such that:

$f(6)=\frac{12}{6}=2 \in \mathbb{Z}$;

this is degree of well-defined.

(ii) There exists the integer $0 \in \mathbb{Z}$, such that:

$f(0)=\frac{12}{0}=$ undefined;

this is degree of indeterminacy.

(iii) There exists the integer, for example $5 \in \mathbb{Z}$, such that:

$f(5)=\frac{12}{5}=2.4 \notin \mathbb{Z}$; or $2.4 \in Q \backslash \mathbb{Z}$;

this is degree of outer-defined.

\subsection{NeutroTheorem}

Let $Z^{+}=\{1,2,3, \ldots,+\infty\}$ be the set of positive integers.

We consider the following:

Statement

If $x$ and $y \in \mathbb{Z}^{+}$, then $x^{y}$ is a perfect square.

In classical algebraic structures this statement is considered false, because it is not $100 \%$ true, and to prove it, it is sufficient to get a single particular counter-example. For example, if $x=2$ and $y=3$, then $2^{3}=8$ which is not a perfect square.

The classical algebraic structures do not leave room for partial truth of a theorem. But, in our world and in our everyday life, we have many more examples of statements that are only partially true, than statements that are totally true.

The NeutroTheorem and AntiTheorem are generalizations and alternatives of the classical Theorem. 
In the above statement, we have:

(i) Degree of truth, when $x=a^{2}$ or $y=2 b$, where $a, b \in \mathbb{Z}^{+}$. Since we get $x^{y}=\left(a^{2}\right)^{y}=\left(a^{y}\right)^{2}$ and respectively $x^{y}=x^{2 b}=\left(x^{b}\right)^{2}$.

Therefore we have two double-infinity many cases when the statement is true.

$\left(3^{4}\right)^{2}=81^{2}$.

$x=a^{2}$ means that $x$ can be written as a perfect square. For example, if $x=3^{8}$ we can re-write it as $x=$

And $y=2 b$ means $y$ is an even number.

(ii) Degree of indeterminacy is zero, since $x^{y}$ is always well-defined for non-zero $x, y \in \mathbb{Z}^{+}$.

(iii) Degree of falsehood, as shown above, for example when $x=2$ and $y=3$.

Herein we also have infinitely many cases when the statement is false (for example when $x \neq a^{2}$ and $y \neq$

$2 b)$

\section{Structure, NeutroStructure, AntiStructure in any field of knowledge}

In general, by NeutroSophication [1, 2, 3, 4], we have extended any classical Structure, in no matter what field of knowledge, to NeutroStructure, and by AntiSophication to AntiStructure.

A classical Structure, in any field of knowledge, is composed of: a non-empty space, populated by some elements, and both (the space and all elements) are characterized by some relations among themselves (such as: laws, operations, operators, axioms, properties, functions, theorems, lemmas, consequences, algorithms, charts, hierarchies, equations, inequalities, etc.), and their attributes (size, weight, color, shape, location, etc.).

\section{Relation, NeutroRelation, AntiRelation}

(i) A classical Relation on a given set is a relation that is true for all elements of the set (degree of truth $\mathrm{T}=1$ ). Neutrosophically we write Relation $(1,0,0)$.

(ii) A NeutroRelation is a relation that is true for some of the elements (degree of truth $\mathrm{T}$ ), indeterminate for other elements (degree of indeterminacy I), and false for the other elements (degree of falsehood F). Neutrosophically we write Relation $(T, I, F)$, where $(\mathrm{T}, \mathrm{I}, \mathrm{F})$ is different from $(1,0,0)$ and from $(0,0,1)$.

(iii) An AntiRelation is a relation that is false for all elements (degree of falsehood F = 1). Neutrosophically we write Relation $(0,0,1)$.

\section{Attribute, NeutroAttribute, AntiAttribute}

(i) A classical Attribute of the elements of a given set is an attribute that is true for all elements of the set (degree of truth $\mathrm{T}=1)$. Neutrosophically we write Attribute $(1,0,0)$.

(ii) A NeutroAttribute is an attribute that is true for some of the elements (degree of truth T), indeterminate for other elements (degree of indeterminacy I), and false for the other elements (degree of falsehood F). Neutrosophically we write Attribute(T,I,F), where (T,I,F) is different from $(1,0,0)$ and $(0,0,1)$.

(iii) An AntiAttribute is an attribute that is false for all elements (degree of falsehood $\mathrm{F}=1$ ). Neutrosophically we write Attribute $(0,0,1)$.

\section{Definitions of Structure, NeutroStructure, AntiStructure}

(i) A classical Structure is a structure whose all elements are characterized by the same given Relationships and Attributes. 
(ii) A NeutroStructure is a structure that has at least one NeutroRelation or one NeutroAttribute, and no AntiRelation nor AntiAttribute.

(iii) An AntiStructure is a structure that has at least one AntiRelation or one AntiAttribute.

\section{Example of NeutroStructure}

In the Christian society the marriage is defined as the union between a male and a female (degree of truth).

But, in the last decades, this law has become less than $100 \%$ true, since persons of the same sex were allowed to marry as well (degree of falsehood).

On the other hand, there are transgender people (whose sex is not well-determined, or whose sex is undetermined), and people who have changed the sex by surgical procedures, and these people (and their marriage) cannot be included in the first two categories (degree of indeterminacy).

Therefore, since we have a NeutroLaw (with respect to the Law of Marriage) we have a Christian NeutroStructure.

\section{Conclusion}

A classical Structure, in any field of knowledge, is composed of: a non-empty space, populated by some elements, and both (the space and all elements) are characterized by some relations among themselves, and by some attributes. Classical Structures are mostly in theoretical, abstract, imaginary spaces.

Of course, when analysing a structure, it counts with respect to what relations and attributes we analyse it.

In our everyday life almost all structures are NeutroStructures, since they are neither perfect nor uniform, and not all elements of the structure's space have the same relations and same attributes in the same degree (not all elements behave in the same way).

\section{References}

[1] F. Smarandache: NeutroAlgebra is a Generalization of Partial Algebra. International Journal of Neutrosophic Science (IJNS), Volume 2, pp. 8-17, 2020. DOI: http://doi.org/10.5281/zenodo.3989285 $\underline{\text { http://fs.unm.edu/NeutroAlgebra.pdf }}$

[2] F. Smarandache, Introduction to NeutroAlgebraic Structures and AntiAlgebraic Structures, in Advances of Standard and Nonstandard Neutrosophic Theories, Pons Publishing House Brussels, Belgium, Chapter 6, pages 240265, 2019; http://fs.unm.edu/AdvancesOfStandardAndNonstandard.pdf

[3] Florentin Smarandache: Introduction to NeutroAlgebraic Structures and AntiAlgebraic Structures (revisited). Neutrosophic Sets and Systems, vol. 31, pp. 1-16, 2020. DOI: 10.5281/zenodo.3638232

http://fs.unm.edu/NSS/NeutroAlgebraic-AntiAlgebraic-Structures.pdf

[4] Florentin Smarandache, Generalizations and Alternatives of Classical Algebraic Structures to NeutroAlgebraic Structures and AntiAlgebraic Structures, Journal of Fuzzy Extension and Applications (JFEA), J. Fuzzy. Ext. Appl. Vol. 1, No. 2, pp. 85-87, 2020. DOI: 10.22105/jfea.2020.248816.1008 http://fs.unm.edu/NeutroAlgebra-general.pdf

[5] A.A.A. Agboola, M.A. Ibrahim, E.O. Adeleke: Elementary Examination of NeutroAlgebras and AntiAlgebras viz-a-viz the Classical Number Systems. International Journal of Neutrosophic Science (IJNS), Volume 4, pp. 1619, 2020. DOI: http://doi.org/10.5281/zenodo.3989530

http://fs.unm.edu/ElementaryExaminationOfNeutroAlgebra.pdf 
[6] A.A.A. Agboola: Introduction to NeutroGroups. International Journal of Neutrosophic Science (IJNS), Volume 6, pp. 41-47, 2020. DOI: http://doi.org/10.5281/zenodo.3989823

http://fs.unm.edu/IntroductionToNeutroGroups.pdf

[7] A.A.A. Agboola: Introduction to NeutroRings. International Journal of Neutrosophic Science (IJNS), Volume 7, pp. 62-73, 2020. DOI: http://doi.org/10.5281/zenodo.3991389

http://fs.unm.edu/IntroductionToNeutroRings.pdf

[8] Akbar Rezaei, Florentin Smarandache: On Neutro-BE-algebras and Anti-BE-algebras. International Journal of Neutrosophic Science (IJNS), Volume 4, pp. 8-15, 2020. DOI: http://doi.org/10.5281/zenodo.3989550

http://fs.unm.edu/OnNeutroBEalgebras.pdf

[9] Mohammad Hamidi, Florentin Smarandache: Neutro-BCK-Algebra. International Journal of Neutrosophic Science (IJNS), Volume 8, pp. 110-117, 2020. DOI: http://doi.org/10.5281/zenodo.3991437

http://fs.unm.edu/Neutro-BCK-Algebra.pdf

[10] Florentin Smarandache, Akbar Rezaei, Hee Sik Kim: A New Trend to Extensions of CI-algebras. International Journal of Neutrosophic Science (IJNS) Vol. 5, No. 1, pp. 8-15, 2020; DOI: 10.5281/zenodo.3788124

http://fs.unm.edu/Neutro-CI-Algebras.pdf

[11] Florentin Smarandache: Extension of HyperGraph to n-SuperHyperGraph and to Plithogenic n-

SuperHyperGraph, and Extension of HyperAlgebra to n-ary (Classical-/Neutro-/Anti-)HyperAlgebra. Neutrosophic Sets and Systems, Vol. 33, pp. 290-296, 2020. DOI: 10.5281/zenodo.3783103

http://fs.unm.edu/NSS/n-SuperHyperGraph-n-HyperAlgebra.pdf

[12] A.A.A. Agboola: On Finite NeutroGroups of Type-NG. International Journal of Neutrosophic Science (IJNS), Volume 10, Issue 2, pp. 84-95, 2020. DOI: 10.5281/zenodo.4277243, http://fs.unm.edu/IJNS/OnFiniteNeutroGroupsOfType-NG.pdf

[13] A.A.A. Agboola: On Finite and Infinite NeutroRings of Type-NR. International Journal of Neutrosophic Science (IJNS), Volume 11, Issue 2, pp. 87-99, 2020. DOI: 10.5281/zenodo.4276366, http://fs.unm.edu/IJNS/OnFiniteAndInfiniteNeutroRings.pdf

[14] A.A.A. Agboola, Introduction to AntiGroups, International Journal of Neutrosophic Science (IJNS), Vol. 12, No. 2, PP. 71-80, 2020. http://fs.unm.edu/IJNS/IntroductionAntiGroups.pdf

[15] M.A. Ibrahim and A.A.A. Agboola, Introduction to NeutroHyperGroups, Neutrosophic Sets and Systems, vol. 38, pp. 15-32, 2020. DOI: 10.5281 /zenodo.4300363, http://fs.unm.edu/NSS/IntroductionToNeutroHyperGroups2.pdf

[16] Elahe Mohammadzadeh and Akbar Rezaei, On NeutroNilpotentGroups, Neutrosophic Sets and Systems, vol. 38, pp. 33-40, 2020. DOI: 10.5281/zenodo.4300370, http://fs.unm.edu/NSS/OnNeutroNilpotentGroups3.pdf 